\title{
Scanner Color Scale
}

National Cancer Institute

\section{Source}

National Cancer Institute. Scanner Color Scale. NCI Thesaurus. Code C94977.

The assignment of a collection of hues to quantitate numerical values being represented in a pixel (voxel) of an image. 